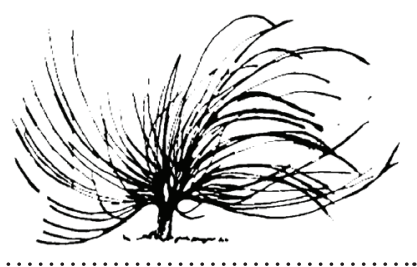

\title{
Aprendizaje autorregulado en la teoría sociocognitiva: Marco conceptual y posibles líneas de investigación
}

\author{
Eduardo Chaves-Barboza ${ }^{1}$ \\ Universidad Nacional \\ Heredia, Costa Rica \\ echav@una.cr
}

\author{
Laura Rodríguez-Miranda ${ }^{2}$ \\ MEP \\ San José, Costa Rica \\ laura.rodriguez.miranda@mep.go.cr
}

\begin{abstract}
Resumen
Este ensayo consiste en una revisión bibliográfica que expone los conceptos de aprendizaje autorregulado y de autorregulación del aprendizaje en el marco de la teoría sociocognitiva. Se hace necesario explicar dos elementos fundamentalmente relacionados: la autoeficacia y la motivación. Además, se explica el vínculo entre la autorregulación del aprendizaje y las competencias que diversos especialistas y organismos internacionales consideran importantes para la formación
\end{abstract}

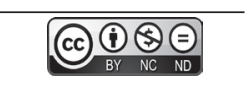

Recibido: 3 de noviembre de 2016-Aprobado: 3 de octubre de 2017

http://dx.doi.org/10.15359/rep.12-2.3

1 Educador y matemático. Ph.D. en Currículo y Formación de Profesorado por la Universidad de Granada, España. Docente desde 1994 en el Ministerio de Educación, la Universidad de Costa Rica y la Universidad Nacional, donde es académico titular desde el 2008 y ha impartido cursos de matemática, investigación, pedagogía y didáctica. Fruto de sus investigaciones ha publicado en revistas especializadas sobre matemática educativa, organización escolar, autorregulación del aprendizaje, entre otros temas.

2 Educadora y humanista. Magíster en Integración de personas con discapacidad por la Universidad de Salamanca, España. Docente de Educación Especial para el Ministerio de Educación Pública desde el 2004, en instituciones de educación primaria y de educación especial. Sus investigaciones versan sobre autorregulación del aprendizaje, docencia y formación del profesorado. Ha servido como voluntaria en instituciones para el desarrollo humano, como Caritas Internationalis y la Pastoral Educativa Católica. 
de los docentes. A lo largo de todo el ensayo se proponen líneas de investigaciones viables, válidas y pertinentes, referidas al aprendizaje autorregulado, algunos conceptos asociados y sus relaciones con otras variables de interés psicopedagógico, en estas propuestas se enfatiza la educación formal y la formación de docentes.

Palabras clave: teoría educacional, formación de docentes, práctica pedagógica, innovación pedagógica.

\begin{abstract}
This essay, a literature review, explains the main concepts of self-regulated learning and self-regulated learning in the context of the social-cognitive theory; it is necessary to explain two elements mainly related to self-regulated learning: self-efficacy and motivation. In addition, this essay explains the relationship between self-regulated learning and the skills that various specialists and international organizations regard as fundamental to the training of teachers. Throughout the entire document, the authors propose viable, valid, and relevant lines of research are proposed based on self-regulated learning, some associated concepts and their relationships with other variables of interest for psychopedagogy. In these proposals, formal education and teacher training are emphasized.
\end{abstract}

Keywords: educational theory, teacher education, teaching practice, pedagogical innovations.

\title{
Introducción
}

La teoría sociocognitiva brinda una perspectiva psicopedagógica del aprendizaje con gran potencial académico. En efecto, conceptos claves de esta teoría, como la motivación, la autorregulación del aprendizaje y la autoeficacia están muy presentes en estudios y reflexiones de reputados equipos de investigación; prueba de ello es su significativa presencia en revistas de alto impacto internacional.

Sin embargo, es lamentable que esta teoría y su influencia en la pedagogía y la psicología sean poco conocidas en Costa Rica. Por 
esta razón, partiendo de una revisión bibliográfica, este ensayo propone líneas de investigación válidas, pertinentes y viables, enfatizando dos grandes e importantes ámbitos del sistema educativo costarricense: la educación formal y los procesos formativos de docentes.

Las líneas de investigación propuestas en este ensayo buscan ampliar los horizontes de investigadores, profesores y estudiantes costarricenses, principalmente aquellos interesados en estudiar temáticas alternativas, desarrollar seminarios de investigación o iniciar trabajos finales de graduación.

\section{Aprendizaje autorregulado en la teoría sociocognitiva}

En la década de los 70 del siglo pasado las teorías educativas comenzaron a centrar su atención en el aprendizaje, en vez de la enseñanza. En 1986 el psicólogo Alberto Bandura desarrolló una teoría sobre el funcionamiento cognitivo humano enfatizando el rol autorregulador y autorreflexivo de la persona cuando tenía que adaptarse a algún cambio. Esto contrastaba con las teorías previas que miraban al sujeto como un ente accionado por al ambiente o que reaccionaba a impulsos internos fuera de su control.

En la década de los 80 del siglo pasado los pedagogos y los psicólogos comienzan a utilizar el término aprendizaje autorregulado desde varias perspectivas teóricas psicopedagógicas, entre ellas, la conductista, la socioconstructivista y la sociocognitiva, iniciando unas corrientes de investigación que se han mantenido con el tiempo (Schunk, 2004; Hadwin et al., 2010; Jin y Low, 2009; Pintrich et al., 1993; Zimmerman, 2008).

En la teoría sociocognitiva el aprendizaje es un proceso mediante el cual el sujeto transforma sus habilidades mentales y creencias epistemológicas en habilidades concretas y conocimientos específicos. En este modelo teórico el individuo que aprende se percibe dentro de un ambiente cambiante que lo obliga a realizar constantes esfuerzos metacognitivos de evaluación y realimentación para ajustar sus conductas y estrategias; consecuentemente, el aprendizaje es visto como una interacción entre elementos intrapersonales, conductuales y ambientales.

En la teoría psicopedagógica sociocognitiva la autorregulación es un proceso motivacional y cognitivo de control, por objetivos 
autodeterminados, mediante el cual la persona busca adaptarse al entorno (Nelson et al., 2015; Carver y Scheier, 1998). En general, la autorregulación de la conducta implica el establecimiento de metas, la evaluación de los objetivos, la reacción emocional al progreso o la falta de progreso y la gestión de recursos hacia la consecución de objetivos, o en su defecto, a la renuncia a los objetivos (Vanthournout et al., 2015; Forgas, Baumeister y Tice, 2009). En consecuencia, en esta teoría, el aprendizaje autorregulado se concibe como un fenómeno donde los individuos sistemáticamente activan y sostienen un proceso cognitivo, motivacional/afectivo y conductual para lograr conocimientos, habilidades y destrezas, de manera eficaz en un determinado contexto (Winne, 2015; Schunk y Zimmerman, 1994; Schunk, 2004; Azevedo, 2007; Zimmerman, 2008).

Para poder continuar con el análisis del aprendizaje autorregulado, es conveniente aclarar en qué consiste la autoeficacia y en qué consiste la motivación dentro de este modelo teórico.

\section{Autoeficacia y motivación en la teoría sociocognitiva}

En la teoría sociocognitiva la eficacia se autorregula, se habla de autoeficacia. La autoeficacia es un constructo teórico que se refiere a las creencias que tiene un individuo sobre su capacidad de realizar las acciones necesarias para alcanzar un objetivo deseado en una determinada situación. La autoeficacia no es una apreciación de si se realizarán estas acciones o si al final se lograrán los resultados deseados, más bien es una autoevaluación de la propia capacidad para efectuar las acciones que se consideran necesarias. Estas creencias no son estables con el tiempo y representan la percepción sobre la propia capacidad en relación con un contexto específico y unos objetivos concretos, esta percepción es una construcción social que es constantemente revisada por el sujeto como resultado de su interacción con otros.

$\mathrm{Al}$ igual que con la autorregulación del aprendizaje, con la autoeficacia se supone que el sujeto tiene un determinado locus de control sobre sus conductas y tareas; enfatiza cómo las personas interactúan e influyen su ambiente, en lugar de ser pasivamente controladas.

Más concretamente, en el caso del estudiante en un sistema de educación formal, la autoeficacia es la creencia del estudiante sobre su propia capacidad de aprender y de cumplir con determinadas tareas 
de algún nivel de dificultad (Bandura, 1977, 1982, 1988; Bandura y Adams, 1977; Maddux, 1995, 2009).

El sujeto aprendiente autoeficaz mira su aprendizaje de forma sistemática y controlable, y acepta su responsabilidad por su rendimiento. Asume sus tareas con confianza, ingenio y dedicación, es proactivo en la búsqueda de información y es activo sobre su propio aprendizaje desde las perspectivas conductual, motivacional y metacognitiva. Los estudiantes con mayor rendimiento académico practican una mayor gama de estrategias de autorregulación y con mayor frecuencia que los demás; es decir, acciones autorregulatorias como la auto-evaluación, determinación de objetivos, planificación de estrategias y registro de seguimiento u observación de actividades y logros (Zimmerman, 2006; Bandura, 2006).

Según Bandura (1997), el individuo tiene percepciones y creencias sobre su propia autoeficacia, y estas percepciones y creencias afectan la forma como alcanza sus logros de aprendizaje autorregulado, influyen sobre la persona para que desee o evite alguna responsabilidad o tarea estudiantil. Percepciones y creencias de elevada autoeficacia (elevado autoconcepto) son necesarias para que el estudiante busque el éxito de forma persistente, activa y motivada; bajo autoconcepto incidirá en que la persona busque evitar las responsabilidades, eventualmente esto la lleva al fracaso y la frustración.

El desarrollar y mantener un sentido de autoeficacia en el estudiante, ayuda a motivar su aprendizaje autorregulado. La autoeficacia se desarrolla con la obtención de éxitos en determinadas tareas, observando cómo otras personas logran éxito en tareas y a través del estímulo y realimentación de sujetos con experiencias de éxito, esto es, en el caso de la educación formal, el docente.

Aquí cabe señalar que el concepto de autoeficacia ofrece prometedoras líneas de investigación en el aprendizaje con tecnologías digitales y en entornos personales de aprendizajes. Porque desde esta teoría puede suponerse una gran influencia sobre el aprendizaje del autoconcepto que tiene el sujeto de su propia eficacia, muy particularmente en su capacidad para seleccionar adecuadas herramientas, para minimizar distracciones y mantener la concentración.

Por su parte, la motivación tiene un lugar especial en la teoría pedagógica sociocognitiva, en ella se define motivación como "el proceso por el cual la actividad intencionada es instigada y sostenida" (Pintrich 
y Schunk, 2002, p. 5). Desde esta perspectiva el modelo sociocognitivo contempla el componente motivacional íntimamente relacionado con la autoeficacia, porque el individuo deriva motivación de la creencia que va a obtener éxito en sus objetivos. Cuando logra éxito en una tarea específica, el sujeto acreciente la confianza en la propia capacidad de realizar tareas asociadas o similares, y esto es una fuente de motivación para nuevas acciones (Pintrich y Schunk, 2002).

En este modelo teórico el aumento en la percepción de la autoeficacia servirá para aumentar la motivación (Zimmerman y Cleary, 2006, 2009). El ayudar a fijar objetivos de aprendizaje, el desarrollo de estrategias para lograr objetivos, el aprendizaje sobre el uso de herramientas de aprendizaje, el mayor control sobre las herramientas que ofrece el entorno de aprendizaje y en general, cualquier destreza, habilidad o conocimiento que permita ampliar la autoeficacia, servirá para incentivar la motivación. Hay incremento de motivación cuando los estudiantes reducen la incertidumbre en el control; es decir, aumentan el control sobre su capacidad de éxito y fracaso, y ven mayor conexión entre sus esfuerzos y el éxito (Covington, 1992; McInerney, 2000; Martin, 2007, 2008, 2009).

En ambientes de educación formal es importante una efectiva orientación y consistente realimentación por parte del profesorado hacia el desarrollo de habilidades y conocimientos para la autorregulación del aprendizaje, esto podría abrir interesantes líneas de investigación. Asimismo, para la motivación del estudiante es clave el uso eficaz de los recursos disponibles, la planificación de estrategias de aprendizaje, la selección de herramientas adecuadas y el desarrollo de un entorno personal de aprendizaje, también estos elementos ofrecen dimensiones para la investigación.

\section{Aprendizaje autorregulado: supuestos teóricos y fases}

En el sociocognitivismo el aprendizaje autorregulado es un constructo teórico fundamentado en cuatro supuestos, el primero consiste en asumir que el sujeto aprendiente es activo en construir sus propios objetivos y significados a partir de su propio sistema cognitivo interno y con la influencia de un determinado entorno.

El segundo es que los individuos son capaces de controlar y dar seguimiento a los elementos cognitivos, motivaciones y conductuales 
de su aprendizaje. El tercero es que la autorregulación está condicionada y es facilitada por factores individuales, como los biológicos, los emocionales y los cognitivos, así como por factores externos, propios del contexto.

Finalmente, se supone que el sujeto tiene la capacidad de evaluar sus propios objetivos de aprendizaje, de monitorear sus conductas y procesos cognitivos y de utilizar los resultados de esta evaluación para regular su propio aprendizaje (Zimmerman, 1990, 2001, 2015; Pintrich, 2004).

Desde este marco conceptual la autorregulación del aprendizaje consiste en un proceso cíclico de tres fases llamadas previsión, actuación y autorreflexión (Zimmerman y Schunk, 1989; Schunk y Zimmerman, 1994, 2003; Boekarts et al., 1999; Pajares, 2002; Pintrich, 1995, 2004; Bandura, 2006; Zimmerman y Cleary, 2006, 2009; Usher y Pajares, 2008; Zimmerman, 2000, 2008, 2015).

En la fase de previsión el individuo plantea sus objetivos de aprendizaje y, partiendo de estos, realiza una planificación estratégica. Por una parte, el sujeto tiene un conjunto de conocimientos previos para efectuar estas acciones. Por otra parte, la realización de estas acciones está muy influida por las percepciones que tiene el sujeto de sí mismo, es decir, de la propia motivación, la propia eficacia y las propias expectativas de aprendizaje. La persona aprendiente no solo debe conocer estrategias para autorregular el aprendizaje, además debe confiar en su propia capacidad para llevar a cabo dichas estrategias exitosamente.

En la fase de actuación, también llamada de control volitivo, se materializan las estrategias de la primera fase mediante conductas, y el sujeto lleva a cabo sus tareas para cumplir con sus objetivos de aprendizaje. Paralelamente, esta fase involucra actuaciones volitivas de autocontrol y de autoobservación, porque el sujeto realiza acciones intencionales de inspección y observación sobre el rendimiento y la eficacia de sus conductas, de las estrategias que está aplicando y del logro que está alcanzando en sus objetivos de aprendizaje.

En la última fase, la autorreflexión, la persona realiza procesos de auto-evaluación y de autojuicio, es decir, utiliza lo observado en la fase anterior y aprovecha sus experiencias previas para realimentarse y tomar decisiones. En esta fase la persona modifica sus conductas, reforma sus estrategias y se prepara para reiniciar el ciclo de autorregulación de sus aprendizajes. 
Como se ha señalado, para el aprendizaje autorregulado, la teoría sociocognitiva supone una persona activa y proactiva, que emplea adaptativamente sus capacidades para realizar tareas estructuradas pero cambiantes en diversos contextos. En las tres fases despliega su iniciativa, su tenacidad y su adaptabilidad para enfocarse, para evitar la procrastinación y, en última instancia, para alcanzar la eficiencia. Las formas como el estudiantado lleva a cabo esto abre oportunidades de investigación.

También, se ha dicho que la percepción de la persona sobre su propia eficacia para alcanzar el éxito en el aprendizaje tiene un papel muy importante en este modelo teórico, porque motiva al individuo a continuar con el ciclo de autorregulación del aprendizaje, y sin motivación, el ciclo se detiene (Nelson et al., 2015; Schunk, 2004; Zimmerman y Cleary, 2006; Pajares, 2007; Usher y Pajares, 2008; Zimmerman, 2008; Jin y Low, 2009). La percepción de la propia eficiencia, es decir, la autoeficacia, implica una conciencia metacognitiva del sujeto sobre las actividades de aprendizaje, las propias conductas, los logros cognitivos y las estrategias elegidas y empleadas (Schunk y Zimmerman, 2003). Tanto la motivación como la autoeficacia son dos variables que abren muchas posibilidades investigativas, pueden interrelacionarse entre ellas y relacionarse con el aprendizaje autorregulado.

\section{Otras líneas investigativas sobre la autorregulación del aprendizaje}

Si bien, el proceso de autorregulación del aprendizaje enfatiza la capacidad gestora de la persona, también es cierto que todo el proceso se lleva a cabo en un entorno social. Es decir, acciones como la regulación de las propias conductas, la monitorización de los logros cognitivos y la adaptación de las estrategias son realizadas bajo la influencia y con la participación de otros sujetos aprendientes, de profesores, de expertos y de toda una red social (Haslaman y Askar, 2015; Rezaee y Mosalanejad, 2015; Nelson et al., 2015). La influencia social sobre el proceso de autorregulación individual es compleja y multidimensional y ofrece desafíos para la investigación; por ejemplo, se puede indagar cómo el individuo muestra sus iniciativas personales y relacionarse con las formas, cómo busca proactivamente la realimentación y el apoyo de sus compañeros, profesores y de su entorno de aprendizaje.

Para Zimmerman (2008) hay cuatro pasos que llevan a un nivel de autorregulación deseada. El primero consiste en observar modelos 
de otros considerados eficientes; el segundo paso es imitar estos modelos mientras recibe la realimentación social. El tercer paso es el autocontrol, que es utilizar, de forma independiente, una habilidad que ha sido demostrada eficaz en una tarea determinada. El cuarto paso es una adecuada autorregulación, que es emplear adaptativamente habilidades para tareas cambiantes en contextos diversos. Estos cuatro pasos brindan dimensiones para investigar la autorregulación del aprendizaje en diversos contextos educativos.

El tema de la autorregulación del aprendizaje ha adquirido mucho auge en entornos de aprendizajes digitales. Estudios como los de Broadbent y Poon (2015), Cheng y Chau (2013), Cho y Cho (2013), Bullock (2013), Holt y Brockett (2012) y Jin y Low (2009) demuestran que los estudiantes que logran autorregular en forma adecuada sus aprendizajes tienden a ser altamente autoeficaces en sus aprendizajes, ser más activos para plantearse y alcanzar objetivos, y reportan menores niveles de procrastinación, que los estudiantes regulados externamente.

Utilizando el marco teórico sociocognitivo es posible iniciar procesos investigativos que expliquen cómo se acceden instantáneamente a casi ilimitadas cantidades de información, cómo evitan perderse en el espacio informático y distraerse con elementos ajenos a sus tareas y responsabilidades. Se puede investigar cómo los sujetos que aprenden asumen diversos roles, como usuarios, clientes, informantes, diseñadores o codiseñadores, entre otros; lo cual amenaza más con descuidar los objetivos de aprendizaje planteados. En efecto, para aprovechar las oportunidades de los recursos digitales e informáticos y superar las amenazas a los objetivos de aprendizaje debe haber un despliegue de habilidades cognitivas, motivacionales y conductuales. La investigación en estas áreas es muy desafiante.

\section{Relación entre la autorregulación del aprendizaje y la formación de docentes}

La capacidad de autorregular los aprendizajes es teóricamente coherente con las competencias que, según criterios de especialistas y organismos internacionales, son fundamentales en la formación de docentes (Aceto et al., 2014; Redecker, 2013; Bullock, 2013; de Hoyos et al., 2013; Green et al., 2013; INTEF, 2013; Parlamento Europeo, 2011; UNESCO, 2011). Esto puede ampliar aún más las posibilidades 
investigativas que ofrece el aprendizaje autorregulado y pone de relieve su importancia y su pertinencia. Estas relaciones teóricas claman ser verificadas en el campo.

\section{Figura 1. Marco de competencias TIC para docentes, UNESCO, 2011}

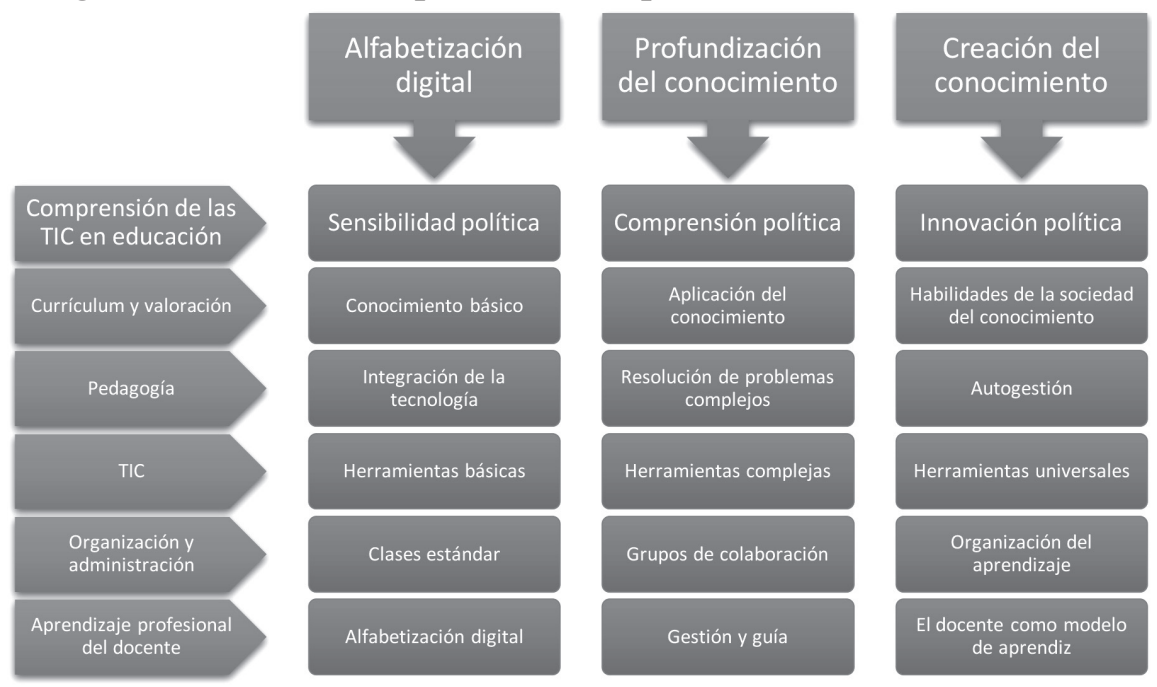

Fuente: UNESCO (2011).

Por ejemplo, la UNESCO (2011), en colaboración con líderes de la industria y expertos mundiales en la formación de profesores, ha establecido las competencias necesarias para que los docentes puedan enseñar con las TIC de manera eficaz. Estas competencias cubren seis aspectos del trabajo del profesor que se colocan en la primera columna de la Figura 1.

Como puede verse en la primera fila de la Figura 1, el marco establecido por la UNESCO plantea para las competencias del docente, tres etapas sucesivas de desarrollo. La primera etapa corresponde a la alfabetización digital, con la que se lograría que los docentes utilicen las TIC para aprender más eficientemente. La segunda etapa es la profundización del conocimiento, en esta etapa el docente adquiere un conocimiento profundo y es capaz de resolver problemas complejos de su profesión y del mundo real. La tercera etapa es la que permite la creación del conocimiento necesario para influir en las decisiones que afectan sus vidas y lograr una sociedad más armoniosa y satisfactoria. 
En este marco, la autorregulación del aprendizaje aparece en doble vía. Por una parte, en el aspecto denominado "Pedagogía" y en la etapa de desarrollo llamada "Creación de conocimiento" (cuarta fila y cuarta columna de la Figura 1), los docentes deben actuar como monitores de los estudiantes para que estos desarrollen las habilidades y los conocimientos que necesitan, informando sobre los métodos para autorregular sus aprendizajes, orientando a los estudiantes para que se integren en comunidades de aprendizaje y cumplan con los plazos acordados para las tareas. Por otra parte, en la última fila y última columna de la Figura 1 se muestra que el docente es un modelo explícito del aprendizaje, esto implica que debe ser capaz de autorregular su propio desarrollo profesional, además de plantear situaciones de aprendizaje para los estudiantes.

Desde esta perspectiva, los docentes son a la vez aprendices, educadores y productores de conocimiento, dedicados a la experimentación e innovación educativas para producir nuevos conocimientos sobre el aprendizaje y la práctica de la enseñanza. También, los docentes deben tener la capacidad, la motivación, la inclinación y el estímulo para experimentar y aprender continuamente, y utilizar las TIC para autorregular su desarrollo profesional en comunidades de aprendizaje profesional. En la Figura 2 se hace referencia al esquema ofrecido por Ala-Mutka (2011, p. 30) en el que se muestran las relaciones entre la alfabetización digital, la alfabetización en TIC y otros conceptos vinculados con el marco del Espacio Europeo de la Educación Superior (EEES) y, por lo tanto, aplican para la formación inicial de docentes.

Dicha esta aclaración, es posible dar la definición de competencia informacional con más claridad. Se entiende por competencia informacional "el conjunto de conocimientos, habilidades, disposiciones y conductas que capacitan a los individuos para reconocer cuándo necesitan información, dónde localizarla, cómo evaluar su idoneidad y darle el uso adecuado de acuerdo con el problema que se les plantea" (Sáinz, 2012, p. 58). Como puede observarse en la Figura 2 la competencia informacional guarda relación con las competencias digitales, en medios de comunicación, en Internet y en TIC. 


\section{Figura 2. Relación entre los alfabetismos digital, informacional, en} Internet, en TIC y en MC*

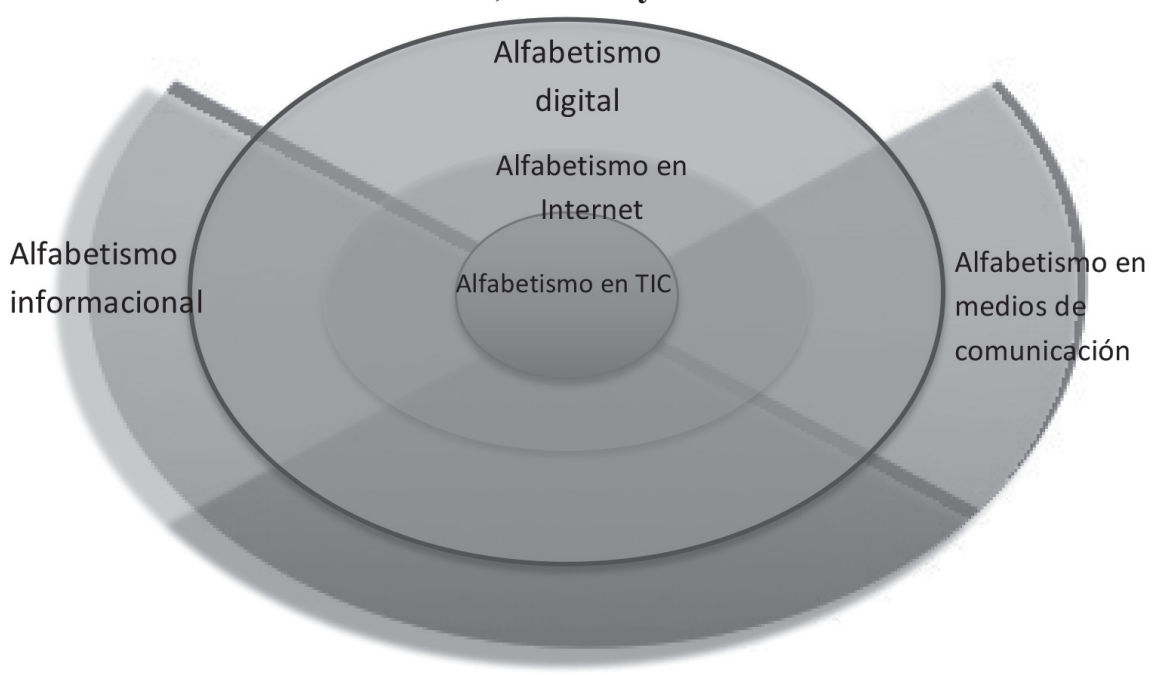

Fuente: Ala-Mutka (2011). *Medios de comunicación.

Esta competencia fue propuesta por la comisión mixta CRUETIC (Conferencia de Rectores de Universidades Españolas) y REBIUN (Red de Bibliotecas Universitarias) para la formación de profesionales en las universidades de España. En particular, la propuesta está completamente vigente en Europa para la formación de los docentes, y busca su completa implementación para el 2020.

Partiendo de esta definición, es evidente la coherencia entre la autorregulación del aprendizaje y la competencia informacional que propone la comisión mixta CRUE-TIC y REBIUN para las universidades españolas. Desde la definición misma la competencia otorga al individuo un claro papel autorregulador de su propia información, y esta relación se hace más notable con las habilidades implicadas en la competencia, estas son (Sáinz, 2012):

1. El estudiante busca la información que necesita.

2. El estudiante analiza y selecciona la información de manera eficiente.

3. El estudiante organiza la información adecuadamente.

4. El estudiante utiliza y comunica la información eficazmente de forma ética y legal, con el fin de construir conocimiento. 
Como puede verse, las habilidades hacen referencia a una autorregulación de la información, y estas habilidades de las personas están íntimamente relacionadas con la autorregulación del aprendizaje. Porque este manejo, ético, personalizado, independiente y eficiente de la información, es necesario para lograr una adecuada autorregulación del aprendizaje, y el desarrollo de la capacidad de autorregulación del aprendizaje permite aumentar las habilidades para autorregular la información.

Por su parte, la competencia digital es "el uso seguro y crítico de las tecnologías de la sociedad de la información para el trabajo, el ocio y la comunicación" (Parlamento Europeo, 2011, p. 7). Como puede verse en la Figura 2 esta competencia se sustenta en las competencias en materia de TIC, e incluye las competencias en Internet.

Las capacidades relacionadas con esta competencia incluyen las siguientes:

La capacidad de buscar, obtener y tratar información, así como de utilizarla de manera crítica y sistemática, evaluando su pertinencia y diferenciando entre información real y virtual, pero reconociendo al mismo tiempo los vínculos. Las personas deben ser capaces de utilizar herramientas para producir, presentar y comprender información compleja y tener la habilidad necesaria para acceder a servicios basados en Internet, buscarlos y utilizarlos, pero también deben saber cómo utilizar las tecnologías de la sociedad de la información en apoyo del pensamiento crítico, la creatividad y la innovación (Parlamento Europeo, 2011, p. 7).

Ala-Mutka (2011) tomó como punto de partida la definición de competencia digital del Parlamento Europeo y del modelo propuesto por el Marco Europeo de Cualificaciones (EQF) 3 para establecer un mapa de los conocimientos, habilidades y actitudes fundamentales para la competencia digital. Este informe fue realizado para el Instituto de Prospectiva Tecnológica del Centro Común de Investigación de la Unión Europea. La propuesta de Ala-Mutka se resume esquemáticamente en la Figura 3.

3 European Qualifications Framework (EQF), su URL es <http://ec.europa.eu/education/ lifelong-learning-policy/eqf_en.htm> 
En este esquema los conocimientos, las habilidades y actitudes se organizan en un orden creciente de complejidad cognitiva, los conocimientos y las habilidades se integraron en dos bloques, en el rojo están los conocimientos y habilidades instrumentales (o básicos) y en el bloque azul, los conocimientos y habilidades superiores (o avanzados). Las actitudes se colocaron separadamente, en el bloque verde.

Como puede observarse, los conocimientos y las habilidades instrumentales corresponden a la alfabetización digital tradicional y se refieren al uso de paquetes ofimáticos y del correo electrónico, la navegación por Internet y la administración de archivos.

En los conocimientos y las habilidades superiores se presentan elementos que resaltan la autorregulación de la información, como el construir un sistema personal para beneficiarse de redes de personas relevantes, el crear una estrategia personal de información con filtros y agentes, y el gestionar identidades digitales específicas para cada situación. Como se explicó anteriormente, estos conocimientos y estas habilidades son indispensables para la autorregulación del aprendizaje y a la vez, el desarrollo de la autorregulación del aprendizaje es fundamental para lograr alcanzar estos conocimientos y habilidades digitales.

Es evidente la importancia de la autorregulación del aprendizaje en la habilidad de encontrar opciones para el aprendizaje personal y profesional. Esta búsqueda de opciones requerirá que el individuo tenga claras sus necesidades de aprendizaje, sea capaz de identificar los recursos humanos y materiales para el aprendizaje, pueda elegir y aplicar estrategias adecuadas de aprendizaje, y evaluar los resultados de sus esfuerzos.

La propuesta de Ala-Mutka (2011) sobre la competencia digital se ha plasmado con especificidad para los docentes en el "Marco común de competencia digital docente" del Plan de Cultura Digital en la Escuela del Instituto Nacional de Tecnologías Educativas y de Formación del Profesorado del MECD (INTEF, 2013). En este marco pueden identificarse descriptores de competencias directamente relacionados con la autorregulación del aprendizaje, entre ellos, el diseño de estrategias para acceder la información y conectarse con comunidades profesionales y redes educativas, la autorregulación de flujos de información y de actividades de colaboración, la evaluación de la utilidad y calidad de recursos educativos disponibles en la WEB. 
La capacidad de autorregulación del aprendizaje es muy pertinente e importante en la formación de docentes y de otros profesionales de la educación, porque este factor activo en la persona aprendiente es el que permite alcanzar el "aprender a aprender" y el "aprendizaje permanente", ambos deseables en la política educativa vigente del EEES (Parlamento Europeo y Consejo Europeo, 2006; Parlamento Europeo, 2011).

Si se concibe aprender a aprender como una de las competencias claves del aprendizaje permanente y se define como "la habilidad para iniciar el aprendizaje y persistir en él, para organizar su propio aprendizaje y gestionar el tiempo y la información eficazmente, ya sea individualmente o en grupos" (Parlamento Europeo y Consejo Europeo, 2006, p. 7). De la misma definición se deduce el peso de la autorregulación del aprendizaje en el aprender a aprender, porque aprender a aprender implica ser conscientes del propio aprendizaje y de las necesidades de aprender por sí mismo, determinando las oportunidades disponibles y superando los obstáculos con el fin de culminar el aprendizaje con éxito.

El aprender a aprender exige: "que la persona gestione eficazmente su aprendizaje, su carrera y su actividad profesional y, en particular, que sea capaz de perseverar en el aprendizaje, de concentrarse en períodos de tiempo prolongados y de reflexionar críticamente sobre los fines y el objeto del aprendizaje" (Parlamento Europeo y Consejo Europeo, 2006, p. 7).

Es decir, el aprender a aprender y aprendizaje permanente implican que las personas sean autónomas, autodisciplinadas y capaces de autorregular su propio aprendizaje. Complementariamente, se espera que la persona sea capaz de trabajar en equipo, de comunicarse en forma asertiva con otras personas, de aprovechar su participación en grupos heterogéneos u homogéneos, de comportarse con ética y sensatez en colectividad y de compartir sus conocimientos.

El aprender a aprender, si bien enfatiza el autorregular eficazmente el aprendizaje, no consiste en un individualismo radical, se refiere a un equilibrado locus de control protagonizado por el individuo y complementado con capacidades interpersonales para comunicarse, compartir y trabajar con otros individuos. Asimismo, no es posible una provechosa interrelación colectiva si los individuos carecen de capacidades que les permita lograr aprendizajes con autonomía, después de todo, el aprendizaje es un proceso intrapsíquico. 


\section{Figura 3. Conocimientos, habilidades y actitudes que contribuyen a la competencia digital}
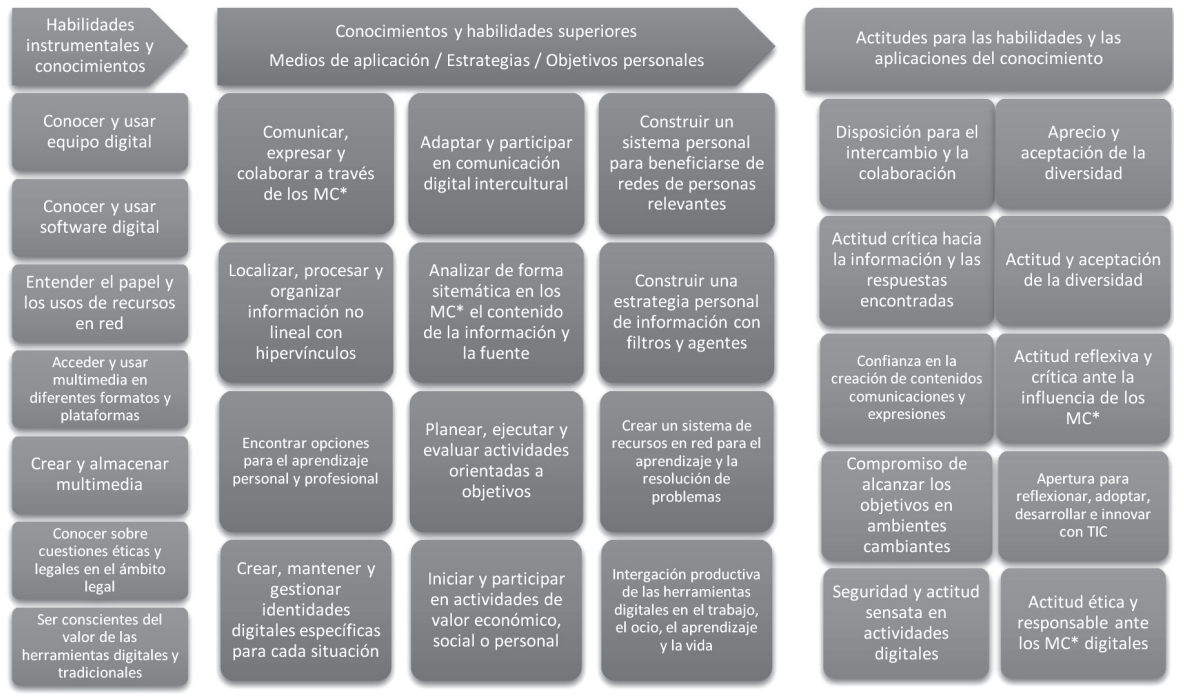

Fuente: Ala-Mutka (2011). *Medios de comunicación.

En síntesis para aprender a aprender "las personas deben ser capaces de organizar su propio aprendizaje, de evaluar su propio trabajo y, llegado el caso, de procurarse asesoramiento, información y apoyo" (Parlamento Europeo y Consejo Europeo, 2006, p. 7) ${ }^{4}$. Esta idea resume la teórica importancia de la autorregulación del aprendizaje para el aprendizaje permanente, la posibilidad de relacionar estas dos variables abre líneas de investigación muy prometedoras.

\section{Conclusiones y recomendaciones}

Derivadas de las reflexiones anteriores se concluye y recomienda:

1. Tanto el aprendizaje autorregulado como algunos de sus conceptos asociados en la teoría sociocognitiva, como la autoeficacia y

4 La conformación de adecuados entornos de aprendizaje ayuda a los estudiantes y docentes en formación a tomar en sus manos el papel central para regular su propio aprendizaje, fijar sus propias metas de aprendizaje personal, organizar sus propios contenidos y procesos de aprendizaje, comunicarse con otros estudiantes y profesores durante los procesos y lograr el cumplimiento de sus objetivos de aprendizaje. 
la motivación, ofrecen posibilidades para la investigación educativa. Las tres son variables que pueden vincularse entre ellas o con otras de interés en la psicopedagogía. Particularmente, es recomendable que se relacionen la motivación y la autoeficacia en futuras investigaciones, para verificar o refutar la teoría existente.

2. En el contexto de la educación formal, la autoeficacia puede relacionarse investigativamente con variables como el éxito escolar, el aprendizaje con tecnologías digitales o la educación en entornos personales de aprendizaje.

3. La motivación puede relacionarse investigativamente con el control del educando sobre el éxito y los esfuerzos propios.

4. En el contexto de la educación formal es pertinente y se recomienda investigar el papel del profesorado en la orientación y la realimentación para motivar la autorregulación del aprendizaje.

5. Durante las fases del aprendizaje autorregulado, pueden sobresalir algunas variables como la iniciativa personal, la tenacidad y la adaptabilidad para enfocarse y evitar la procrastinación. Se recomienda fomentar líneas de investigación en este sentido.

6. Se recomienda que futuras líneas de investigación, en diversos contextos educativos, se refieran a la influencia de distintos actores del entorno educativo sobre la autorregulación del aprendizaje. También es recomendable que se refieran a los pasos de Zimmerman (2008) para una autorregulación deseada, a la autorregulación del aprendizaje en entornos personales educativos, o bien a las habilidades cognitivas y conductuales necesarias para la autorregulación del aprendizaje con apoyo de tecnologías informáticas.

7. La relación teórica que existe entre la capacidad de autorregular los aprendizajes y las competencias fundamentales vigentes para la formación de docentes debe ser verificada mediante procesos de investigación. Se recomienda verificar la relación entre el aprendizaje permanente (Ala-Mutka, 2011) y la autorregulación del aprendizaje. 


\section{Referencias}

Aceto, S., Borotis, S., Devine, J. y Fischer, T. (2014). Results from a consultation with European stakeholders and roadmaps for policy action [Resultados de una consulta con las partes interesadas europeas y hojas de ruta para la acción política]. In P. Kampylis \& Y Punie (Eds.), Mapping and Analysing Prospective Technologies for Learning. Luxembourg: Publications Office of the European Union. Recuperado de $<$ http://publications.jrc.ec.europa. eu/repository/handle/JRC88469>

Ala-Mutka, K. (2011). Mapping Digital Competence: Towards a Conceptual Understanding [Mapeo de la Competencia Digital: Hacia un Entendimiento Conceptual]. Luxembourg: Publications Office of the European Union. Recuperado de http://www.jrc. ec.europa.eu

Azevedo, R. (2007). Understanding the complex nature of self-regulatory processes in learning with computer-based learning environments: an introduction [Comprender la naturaleza compleja de los procesos de autorregulación en el aprendizaje con entornos de aprendizaje basados en computadoras: una introducción. $M e-$ tacognition Learning, 2, 57-65.

Bandura A. (1977). Self-efficacy: Toward a unifying theory of behavioral change. [Autoeficacia: Hacia una teoría unificadora del cambio de comportamiento]. Psychological Review, 84, 191-215.

Bandura, A. (1982). Self-efficacy mechanism in human agency [Mecanismo de autoeficacia en la agencia humana]. American Psychologist, 37, 122-147.

Bandura, A. (1988). Self-efficacy conception of anxiety [Concepto de autoeficacia de la ansiedad]. Anxiety, Stress, and Coping, 1, 77-98.

Bandura, A. (1997). Self-efficacy: The exercise of control [Autoeficacia: El ejercicio del control]. New York: Freeman.

Bandura, A. (2006). Guide for constructing self-efficacy scales [Guía para construir escalas de autoeficacia.] In F. Pajares \& T. Urdan (Eds.), Adolescence and education [Adolescencia y educación] (Self- efficacy and adolescence, Vol. 5, pp. 307-337). Greenwich, CT: Information Age Publishing. 
Bandura, A. y Adams, N. E. (1977). Analysis of self-efficacy theory of behavioral change. [Análisis de la teoría de autoeficacia del cambio de comportamiento.] Cognitive Therapy and Research, 1, 287-308.

Boekarts, M., Pintrich, P. y Zeidner, M. (Eds.). (1999). Handbook of self-regulation [Manual de autorregulación]. New York: Academic.

Broadbent, J. y Poon, W. L. (2015). Self-regulated learning strategies $\&$ academic achievement in online higher education learning environments: A systematic review [Estrategias de aprendizaje auto-reguladas y logros académicos en entornos de aprendizaje en línea de educación superior: Una revisión sistemática]. The Internet and Higher Education. doi:10.1016/j.iheduc.2015.04.007

Bullock, S. M. (2013). Using digital technologies to support Self-Directed Learning for preservice teacher education [Uso de tecnologías digitales para apoyar el Aprendizaje Autodirigido]. Curriculum Journal, 24(1), 103-120. doi:10.1080/09585176.2012.744695

Carver, C. S. y Scheier, M. F. (1998). On the Self-Regulation of Behavior [Sobre la autorregulación de la conducta]. New York, NY: Cambridge University Press.

Cheng, G. y Chau, J. (2013). Exploring the relationship between students' self-regulated learning ability and their ePortfolio achievement [Explorar la relación entre la capacidad de aprendizaje auto-regulado de los estudiantes y su logro de ePortfolio]. The Internet and Higher Education, 17, 9-15. doi:10.1016/j. iheduc.2012.09.005

Cho, K. y Cho, M. H. (2013). Training of self-regulated learning skills on a social network system [Capacitación de habilidades de aprendizaje autorreguladas en un sistema de redes sociales]. Soc Psychol Educ, 16(4), 617-634. doi:10.1007/s11218-013-9229-3

Covington, M. V. (1992). Making the grade: A self-worth perspective on motivation and school reform [Hacer el grado: Una perspectiva de autoestima sobre la motivación y la reforma escolar]. Cambridge: Cambridge University Press.

de Hoyos, M., Green, A. E., Barnes, S. A., Behle, H., Baldauf, B. y Owen, D. (2013). ICT and Employability. In C. Centeno \& J. Stewart (Eds), Literature Review on Employability, Inclusion and ICT, Report 2 [Revisión de Literatura sobre Empleabilidad, 
Inclusión y TIC, Informe 2]. Luxembourg: Publications Office of the European Union. Recuperado de http://is.jrc.ec.europa.eu/ pages/Publications.html

Forgas, J. P., Baumeister R. F. y Tice D. M. (2009). Psychology and Self-Regulation: Cognitive, Affective, and Motivational Processes [Psicología y Autorregulación: Procesos Cognitivos, Afectivos y Motivacionales]. New York, NY: Psychology Press.

Green, A. E., de Hoyos, M., Barnes, S. A., Owen, D., Baldauf, B. y Behle, H. (2013). The Concept of Employability with a Specific Focus on Young People, Older Workers and Migrants [El concepto de empleabilidad con un enfoque específico en los jóvenes, los trabajadores mayores y los migrantes]. In C. Centeno \& J. Stewart (Eds), Literature Review on Employability, Inclusion and ICT, Report 1 [Revisión de Literatura sobre Empleabilidad, Inclusión y TIC, Reporte 1]. Luxembourg: Publications Office of the European Union. Recuperado de http://is.jrc.ec.europa.eu/ pages/Publications.html

Hadwin, A. F., Oshige, M., Gress, C. L. Z. y Winne, P. H. (2010). Innovative ways for using gStudy to orchestrate and research social aspects of self-regulated learning [Maneras innovadoras de utilizar gStudy para orquestar e investigar los aspectos sociales del aprendizaje autorregulado]. Computers in Human Behavior, 26(5), 794-805.

Haslaman, T. y Askar, P. (2015). The Measures of Students' Self-Regulated Learning and Teachers' Supportive Self-Regulated Learning Behaviors [Las medidas del aprendizaje auto-regulado de los estudiantes y los comportamientos de aprendizaje auto-regulados de apoyo de los maestros]. Hacettepe University Journal of Education, 30(1), 106-121.

Holt, L. y Brockett, R. G. (2012). Self-direction and factors influencing technology use: Examining the relationships for the 21st century workplace [Autodirección y factores que influyen en el uso de la tecnología: Examinar las relaciones para el lugar de trabajo del siglo XXI]. Computers in Human Behavior, 28(6), 2075-2082. doi:10.1016/j.chb.2012.06.011

INTEF (2013). Marco común de Competencia Digital Docente del Plan de Cultura Digital en la Escuela. España: Instituto Nacional de Tenologías Eductivas y de Formación del Profesorado (MEP). 
Recuperado de http://educalab.es/documents/10180/12809/MarcoComunCompeDigiDoceV2.pdf

Jin, P. y Low, R. (2009). Enhancing motivation and self-regulated learning in multimedia environments [Mejorar la motivación y el aprendizaje autorregulado en entornos multimedia.] In R. D. Koo, B. C. Choi, M. R. D. Lucas, \& T. C. Chan (Eds.), Education policy, reform, and school innovations in the Asia-Pacific Region (pp. 525-547). Hong Kong: Association for Childhood Education International - Hong Kong \& Macao (ACEI-HKM).

Maddux, J. E. (ed.) (1995). Self-Efficacy, Adaptation, an Adjustment: Theory, Research, and Application [Autoeficacia, Adaptación, Ajuste: Teoría, Investigación y Aplicación]. New York: Plenum.

Maddux, J. E. (2009). Self-efficacy: The power of believing you can. [Self-efficacy: The power of believing you can]. In S. J. Lopez \& C. R. Snyder (Eds.), Oxford Handbook of Positive Psychology, 2nd ed. (pp. 335-343). New York: Oxford University Press.

Martin, A. J. (2007). Examining a multidimensional model of student motivation and engagement using a construct validation approach [Examinar un modelo multidimensional de motivación y compromiso estudiantil utilizando un enfoque de validación de constructos]. British Journal of Educational Psychology, 77, 413-440.

Martin, A. J. (2008). Enhancing student motivation and engagement: The effects of a multidimensional intervention [Mejorar la motivación y la participación de los estudiantes: Los efectos de una intervención multidimensional]. Contemporary Educational Psychology, 33, 239-269.

Martin, A. J. (2009). The motivation and engagement scale - User manual (9th ed.) [La escala de motivación y compromiso - Manual del usuario (9th ed.)]. Sydney: Lifelong Achievement Group. www. lifelong achievement.com.

McInerney, D. (2000). Helping kids achieve their best [Ayudar a los niños a lograr lo mejor]. Sydney: Allen and Unwin.

Nelson, K. G., Shell, D. F., Husman, J., Fishman, E. J. y Soh, L. K. (2015). Motivational and Self-Regulated Learning Profiles of Students Taking a Foundational Engineering Course [Perfiles de aprendizaje motivacional y autorregulado de los estudiantes que toman un curso de ingeniería fundacional]. Journal of Engineering Education, 104(1), 74-100. 
Pajares, F. (2002). Gender and perceived self-efficacy in self-regulated learning [Género y autoeficacia percibida en el aprendizaje autorregulado]. Theory into Practice, 41, 116-225.

Pajares, F. (2007). Motivational role of self-efficacy beliefs in self- regulated learning [Rol de motivación de las creencias de autoeficacia en el aprendizaje autorregulado]. In B. J. Zimmerman \& D. H. Schunk (Eds.), Motivation and self-regulated learning: Theory, research, and applications (pp. 111-140). New York: Erlbaum.

Parlamento Europeo. (2011). Resolución del Parlamento Europeo, de 18 de mayo de 2010, sobre competencias clave para un mundo cambiante: Puesta en práctica del programa de trabajo «Educación y Formación 2010» (2010/2013(INI)). Diario oficial de la Unión Europea número 2011/C 161 E/8 del 31 de mayo de 2011. Recuperado de http://eur-lex.europa.eu/

Parlamento Europeo y Consejo Europeo. (2006). Recomendación del Parlamento Europeo y del Consejo de 18 de diciembre de 2006 sobre las competencias clave para el aprendizaje permanente. Diario oficial de la Unión Europea número 2006/L 394/10 del 30 de diciembre de 2006. Disponible en $<$ http://eur-lex.europa.eu/ $>$.

Pintrich, P. (Ed.). (1995). Understanding self-regulated learning [Comprender el aprendizaje autorregulado]. San Francisco: Jossey-Bass.

Pintrich, P. R. (2004). A conceptual framework for assessing motivation and self-regulated learning in college students [Un marco conceptual para evaluar la motivación y el aprendizaje autorregulado en los estudiantes universitarios]. Educational Psychology Review, 16(4), 385-407.

Pintrich, P. y Schunk, D. (2002). Motivation in education: Theory, research, and application (2nd ed.) [Motivación en la educación: teoría, investigación y aplicación (2nd ed.)]. Upper Saddle River: Merrill Prentice Hall

Pintrich, P. R., Smith, D., Garcia, T. y McKeachie, W. (1993). Predictive validity and reliability of the Motivated Strategies for Learning Questionnaire (MSLQ) [Validez predictiva y confiabilidad del cuestionario Motivated Strategies for Learning Questionnaire (MSLQ)]. Educational and Psychological Measurement, $53,801-813$. 
Redecker, C. (2013). The use of ICT for the assessment of key competences [El uso de las TIC para la evaluación de las competencias clave]. Luxembourg: Publications Office of the European Union. Recuperado de http://is.jrc.ec.europa.eu/pages/Publications.html

Rezaee, R. y Mosalanejad, L. (2015). The effects of case-based team learning on students' learning, self regulation and self direction [Los efectos del aprendizaje en equipo basado en casos sobre el aprendizaje de los estudiantes, la autorregulación y la auto dirección]. Glob J Health Sci, 7(4), 295-306. doi:10.5539/gjhs. $\mathrm{v} 7 \mathrm{n} 4 \mathrm{p} 295$

Sáinz, R. M. (Ed.) (2012). Universidad 2020: Papel de las TIC en el nuevo entorno socioeconómico. Madrid, España: Col. Editorial Ariel, S.A., Fundación Telefónica y Editorial Planeta.

Schunk, D. H. (2004). Learning theories: An educational perspective (4th ed.) [Teorías del aprendizaje: una perspectiva educativa (4th ed.)]. Upper Saddle River: Pearson Education.

Schunk, D. H. y Zimmerman, B. J. (Eds.). (1994). Self-regulation of learning and performance [Autorregulación del aprendizaje y el rendimiento]. Hillsdale: Lawrence Erlbaum.

Schunk, D. H. y Zimmerman, B. J. (2003). Self-regulation and learning [Autorregulación y aprendizaje]. In W. M. Reynolds \& G. E. Miller (Eds.), Handbook of psychology (Vol. 7): Educational psychology (pp. 59-78). Hobo- ken, NJ: Wiley.

UNESCO. (2011). The UNESCO Information and Communication Tools Competency Framework for Teachers (UNESCO ICT CFT) [El Marco de Competencia de las Herramientas de Información y Comunicación de la UNESCO para los Docentes (ICT ICT de la UNESCO)]. París: United Nations Educational, Scientific and Cultural Organization Publications.

Usher, E. L. y Pajares, F. (2008). Self-efficacy for self-regulated learning: A validation study [Autoeficacia para el aprendizaje autorregulado: un estudio de validación]. Educational and Psychological Measurement, 68, 443-463.

Vanthournout, G., Kyndt, E., Gijbels, D. y Van den Bossche, P. (2015). Understanding the direct and indirect relations between motivation to participate, goal orientation and the use of self-regulation strategies during a formal training [Comprender las relaciones directas e indirectas entre la motivación para participar, la 
orientación a las metas y el uso de estrategias de autorregulación durante una formación formal]. Z Erziehungswiss, 18, 89-106. doi:10.1007/s11618-014-0601-7

Winne, P. H. (2015). Self-Regulated Learning [Aprendizaje auto-regulado]. In J. D. Wright (Ed.), International Encyclopedia of the Social \& Behavioral Sciences (pp. 535-540). Oxford: Elsevier. doi:10.1016/B978-0-08-097086-8.25091-5

Zimmerman, B. J. (1990). Self-regulated learning and academic achievement: an overview [Aprendizaje autorregulado y rendimiento académico: una visión general]. Educational Psychologist, 25, 3-17.

Zimmerman, B. J. (2000). Attaining self-regulation: A social cognitive perspective [Lograr la autorregulación: una perspectiva social cognitiva]. In M. Boekaerts, P. R. Pintrich, \& M. Zeidner (Eds.), Handbook of self-regulation (pp. 13-39). San Diego: Academic Press.

Zimmerman, B. J. (2001). Theories of self-regulated learning and academic achievement: an overview and analysis [Teorías del aprendizaje auto-regulado y logro académico: una visión general y análisis]. In B. J. Zimmerman, y D. E. Schunk (Eds.), Self-regulated learning and academic achievement: Theoretical perspectives (pp. 1-37). Mahwah, NJ: Erlbaum.

Zimmerman, B. J. (2006). Self-regulation and effective learning. [Autorregulación y aprendizaje efectivo]. In: D.M. McInerney \& V. McInerney (Eds.), Educational psychology: Constructing learning (pp. 190-191). Sydney: Pearson Education.

Zimmerman, B. J. (2008). Investigating self-regulation and motivation: Historical background, methodological development, and future prospects [Investigación de la autorregulación y motivación: Antecedentes históricos, desarrollo metodológico y perspectivas futuras]. American Educational Research Journal, 45(1), 166-183.

Zimmerman, B. J. (2015). Self-Regulated Learning: Theories, Measures, and Outcomes [Aprendizaje auto-regulado: teorías, medidas y resultados]. In J. D. Wright (Ed.), International Encyclopedia of the Social \& Behavioral Sciences (pp. 541-546). Oxford: Elsevier. doi:10.1016/B978-0-08-097086-8.26060-1

Zimmerman, B. J. y Cleary, T.J. (2006). Adolescents' development of personal agency. [El desarrollo de la agencia personal por parte de los adolescentes]. In F. Pajares \& T. Urdan (Eds.), Adolescence 
and education (Self-efficacy beliefs of adolescents, Vol. 5, pp. 45-69) [Adolescencia y educación (Creencias de autoeficacia de adolescentes, Vol. 5, pp. 45-69)]. Greenwich, CT: Information Age Publishing.

Zimmerman, B.J. y Cleary, T.J. (2009). Motives to self-regulate learning [Motivos para auto-regular el aprendizaje]. In K. R. Wentzel \& A. Wigfield (Eds.), Handbook of motivation at school (pp. 247-264). New York, NY: Routledge.

Zimmerman, B. J. y Schunk, D. H. (Eds.). (1989). Self-regulated learning and academic achievement: Theory, research and practice [Aprendizaje y rendimiento académico autorregulados: Teoría, investigación y práctica]. New York: Springer. 